\title{
Age-related changes in longitudinal prestress in human abdominal aorta
}

\author{
Lukas Horny $^{1 *}$, Tomas Adamek ${ }^{2}$, Rudolf Zitny ${ }^{1}$ \\ ${ }^{1}$ Faculty of Mechanical Engineering, Czech Technical University in Prague \\ Technicka 4, 166 07, Prague, Czech Republic \\ 2 Third Faculty of Medicine, Charles University in Prague, \\ Ruska 87, 100 00, Prague, Czech Republic \\ * Corresponding author: tel.: +420 224352 690, fax: +420 233322 482, \\ email: lukas.horny@fs.cvut.cz
}

\begin{abstract}
Studies on the influence of aging on the longitudinal mechanical response of elastic arteries are rare, though longitudinal behavior may have a significant effect on pressure pulse transmission. Our study was designed to elucidate how aging is reflected in changes of the longitudinal prestress, prestretch and pretension force. The study involved ten human samples (six female, four male) of the abdominal aorta with longitudinal prestretch determined in autopsy. Cylindrical samples underwent a longitudinal elongation test in order to estimate the force necessary to attain the in situ length and to determine the corresponding axial prestress. The elastic modulus was estimated employing hyperelastic limiting chain extensibility model. It was found that pretension force, longitudinal prestress and prestretch are negatively correlated with age. The decreased longitudinal force necessary to obtain the in situ length suggested that the decrease in the prestress occurs not only due to the age-related increase in the crosssection area. Since elastin is the main constituent responsible for bearing the prestretch, this suggests that the observed decrease in the longitudinal prestress and prestretch reflects aging-induced damage to the elastin. Finally, constitutive modeling showed that limiting chain extensibility is a concept that is suitable for describing the aging effect.
\end{abstract}

\section{Keywords}

Abdominal aorta; Aging; Arteriosclerosis; Biomechanics; Elasticity; Prestrain. 


\section{Introduction}

Elastic arteries in situ are significantly prestretched in axial direction; they retract upon excision [1,2]. This phenomenon, although well-known, has been rarely studied in detail - especially human data can be found in only limited number of reports. Nevertheless, axial prestress in an artery has important physiological function. In idealized case, it enables the artery to carry the pressure pulse with minimal variation in its length [3-5].

Recent studies have proven longitudinal prestretch to be a subject of active arterial remodeling. Axial overstretching led to cell proliferation and matrix synthesis to adapt arteries to changed mechanical environment [6-9]. Lengthening of endothelial cells [10] as well as lengthening of unloaded artery segment length [11] were also observed.

Ex vivo mechanical tests with enzymatically treated arteries $[12,13]$ and genetically modified animal models $[14,15]$ suggest crucial role of elastin for bearing the prestretch. The hypothesis of fundamental elastin's function is in accordance with distally increasing magnitude of the prestretch $[2,16]$. Caudally decreasing number of elastin lamellae, which sustain approximately constant axial force in the whole aorta [17], lead to increased force per lamella which results in increasing prestretch.

In this paper, we continue with a study of the longitudinal mechanical behavior of human abdominal aorta, started with a detailed description of age-related changes in the longitudinal prestretch and its correlation with the diameter and with atherosclerosis described in $[1,18,19]$. Previous autopsy measurements of the prestretch have shown a nonlinear age-related decrease in the prestretch magnitude accompanied by a nonlinear increase in the aortic diameter. They, however, have not revealed the magnitudes of the corresponding longitudinal pretension force and prestress.

\section{Methods}

Here we present an experiment aimed at determining the effect of aging on the force that preloads the aorta in the axial direction (the force needed to attain in situ length) and the corresponding axial prestress. The experimental data is used in the constitutive modeling to elucidate age-dependent changes in stiffness related to longitudinal prestretch. Since autopsy measurement (excision from a body) is the only direct method available for detecting non-prestretched length, a simple elongation test of tubular samples was used. To mimic the conditions of autopsy measurement of in situ and ex situ length, the aortas were not pressurized in these tests. 


\subsection{Experiment}

Prestretch. Longitudinal prestretch of the aorta was quantified by ratio (1). Here $l$ denotes the length of the artery segment in situ and $L$ denotes the length after excision from the body. The prestretch was measured in each sample in the way described in [1,19].

$$
\lambda_{\text {AUTOPSY }}=\frac{l}{L}
$$

Pretension force and mechanical response. The experiments were performed with samples of human infrarenal aorta obtained from regular autopsies. The Ethical Committee of the Third Faculty of Medicine of the Charles University in Prague approved the use of human tissue in this research. After being transported to the laboratory, the samples were equilibrated to the laboratory temperature $\left(22^{\circ} \mathrm{C}\right)$. Then they were cannulated at both ends, marked with a liquid eyeliner and suspended on a stand. Each cylindrical sample was consecutively elongated by a longitudinal load up to at least a force of $1.6 \mathrm{~N}$. The specimen was photographed in each loading step, and the longitudinal stretch $\lambda_{z Z}$ was determined through image analysis as the ratio of the deformed to the reference distance between marks on the specimen.

Each experiment consisted in at least fifteen loading steps, which spanned approximately three minutes. To avoid a time-dependent material response (creep), the specimen was unloaded between each pair of longitudinal weights. The average loading time in each step was approximately $5 \mathrm{~s}$.

The experimental Cauchy stress $\sigma_{z z}^{E X P}$ was determined by (2), where $F$ is the acting axial force and $S$ is the reference cross-sectional area given as the product of the circumference and the thickness of the sample. Incompressibility of the tissue was assumed (no volume change during a deformation).

$$
\sigma_{Z Z}^{E X P}=\lambda_{z Z} \frac{F}{S}
$$

\subsection{Model}

Stress-strain relationship and elastic modulus. The hyperelastic limiting chain extensibility model $[20,21]$ was employed to determine the longitudinal stiffness of the prestretched aorta. It is a two-parametric model belonging to the class of so-called generalized neo-Hookean models, which is able to describe large strain stiffening (3) and its successful use in arterial mechanics has been reported in the literature $[21,49,50]$.

$$
W=-\frac{\mu J_{m}}{2} \ln \left(1-\frac{I_{1}-3}{J_{m}}\right)
$$


$W$ denotes the strain energy density function; $\mu$ is the infinitesimal shear modulus (a stress-like parameter corresponding to the initial stiffness); and $J_{\mathrm{m}}$ is a (dimensionless) limiting extensibility parameter which governs the rate of stiffening. $I_{1}$ is the first invariant of the left Cauchy-Green strain tensor $\mathbf{b}\left(I_{1}=\operatorname{tr}(\mathbf{b})\right)$ computed from the deformation gradient $\mathbf{F}, \mathbf{b}=\mathbf{F} \mathbf{F}^{\mathrm{T}}$.

The assumption of incompressibility of the artery wall was also adopted. It implies $I_{3}=\operatorname{det}^{2}(\mathbf{F})=$ $\operatorname{det}(\mathbf{b})=1$. Constitutive equation, i.e. stress-strain relationship, for a hyperelastic material is obtained via differentiation of $W$ with respect to $\mathbf{b}(4)$.

$$
\boldsymbol{\sigma}=2 \frac{\partial W(\mathbf{b})}{\partial I_{1}} \mathbf{b}-p \mathbf{I}
$$

Here $p$ accounts for the reaction to the incompressibility constraint, and will be determined from a boundary condition. The final form of the Cauchy stress $\sigma$ is written in equation (5). I denotes a unit second order tensor.

$$
\boldsymbol{\sigma}=\mu \frac{J_{m}}{J_{m}-\left(I_{1}-3\right)} \mathbf{b}-p \mathbf{I}
$$

Our study is focused on determining the mechanical state of the aorta corresponding to the prestretch found in an autopsy. We note that before and during an autopsy excision of the aorta no blood pressure is acting in the body. Thus, an experiment aimed to find the force necessary to extend an arterial segment to in situ dimensions cannot include pressurization.

It was assumed that the longitudinal force deforms a cylindrical segment of aorta to an elongated cylinder with decreased radius. The reference cylindrical coordinates $(R, \Theta, Z)$ are mapped on to the deformed coordinates $(r, \theta, z)$ by means of the equations $r=r(R), \theta=\Theta, z=\lambda Z$ (here $\lambda_{z Z}=\lambda$ ). The longitudinal stretch $\lambda$ is considered to be uniform along the length of the sample. Since no transmural pressure is acting, the contribution of the collagen fibers (the main source of arterial anisotropy) to the load-carrying process is considered to be negligible. In this situation, the mechanical response is dominated by elastin and is modeled as isotropic [21-23]. Now, incorporating the thin-walled approximation, $\mathbf{F}$ is obtained (6).

$$
\mathbf{F}=\left(\begin{array}{ccc}
\lambda_{r R} & 0 & 0 \\
0 & \lambda_{\theta \Theta} & 0 \\
0 & 0 & \lambda_{z Z}
\end{array}\right)=\left(\begin{array}{ccc}
\lambda^{-\frac{1}{2}} & 0 & 0 \\
0 & \lambda^{-\frac{1}{2}} & 0 \\
0 & 0 & \lambda
\end{array}\right)
$$

The boundary condition necessary to complete the stress-strain relationship by $p$ is adopted as $\sigma_{r r}$ $=0$. The model then results in equation (7) which describes the longitudinal stress acting in the artery extended along the direction of its axis at no internal pressure $\left(I_{1}=\lambda^{2}+2 / \lambda\right)$. 


$$
\sigma_{z z}^{M O D}=\mu \frac{J_{m}}{J_{m}-\left(I_{1}-3\right)}\left(\lambda^{2}-\frac{1}{\lambda}\right)
$$

The elastic modulus is the slope of the tangent to a stress-strain curve. Fitted models (3) were employed to this end rather than experimental stress-strain data. In the present case, the general approach via the elasticity (sometimes called stiffness) tensor was used. It is obtained by differentiating a stress with respect to a strain. The result is a fourth-order tensor $\underline{\mathbf{c}}$, where the components are functions of the specific strain state and the material parameters (8).

$$
\underline{\mathbf{c}}=2 \mathbf{b} \frac{\partial \boldsymbol{\sigma}}{\partial \mathbf{b}}
$$

Substituting $\sigma_{z z}^{M O D}$ into (8), we will approach towards (9). A numerical value for the elastic modulus of the material is then attained after substituting specific $\lambda_{\text {AUTOPSY }}$ into (9).

$$
\underline{c}_{z z z z}=\mu \frac{J_{m}}{J_{m}-\left(I_{1}-3\right)} \frac{1}{\lambda}\left(\frac{2\left(1-\lambda^{3}\right)^{2}}{\lambda\left(J_{m}-\left(I_{1}-3\right)\right)}+3\right)
$$

Correlation with age. All studied quantities and model parameters were involved in the correlation analysis. The linear correlation coefficient $R$ is supplemented by hypothesis testing. Two kinds of tests were employed. The first was a $t$-test with the null hypothesis $\mathrm{H}_{0}: R=0$ ( $p$-value denoted $p_{R}$ ). The second was a $t$-test related to the efficiency of the linear regression model with the null hypothesis $\mathrm{H}_{0}$ : $a=0$ (in the regression equation $y=a x+b ; p_{a}$ ). The power law was also used. In this case, the null hypothesis states $\mathrm{H}_{0}: b=0$ (considering equation $y=a x^{b}$ and its logarithmic transformation; $p_{b}$ ). The results are considered to be significant at $\alpha=0.05$.

Sample size effect, and loss of pretension. Our previous studies reporting statistics for the longitudinal prestretch in human abdominal aorta included 250 samples (60 female, 190 male; [18]). The current study involved only 10 samples, which could lead to biased results. Current data was compared with previous observations to exclude potential outliers. Moreover, incorporation of the previous data, with supplementary presumptions, provided a more reliable basis for estimating prestretch-age, pretension force-age, and prestress-age relationships. In what follows, we will distinguish between the small data sample (10 specimens with full experimental record, Table 1) and the large data sample (250 prestretches - specific values in online supplement of this document).

All the data was normalized with respect to the maximum value, which was presumed to appear at the age of 20 years. A decreasing trend in prestretch with increasing age was observed in previous analyses, and it is reasonable to expect this trend also in the relationships between pretension forceage and prestress-age. In addition to the simultaneous occurrence of the maximum value, we also postulated that the minimum value will be reached at the same time. The prestretch was substituted by 
the engineering prestrain $\varepsilon(=\lambda-1)$ and the minimum value for studied quantities was prescribed to zero.

A zero value is justified by the following considerations. First, zero-prestretched arteries were observed (they do not retract upon excision). Second, it is well known that human abdominal aorta may form an aneurysm (AAA), which can be considered as a manifestation of the loss of longitudinal prestretch. Better to say, the loss of prestretch can be accompanied by the formation of AAA, because loss of pretension also exists in another form. The other form is so-called tortuosity (loss of straightness of the vessel axis). It means that the aorta does not expand radially (as in AAA), but longitudinally. However, due to fixation at the ends of the infrarenal segment, the aorta buckles laterally.

The zero-prestretch time was prescribed at 85 years of age. This value was estimated from statistics reporting the prevalence of aneurysms in the population. The prevalence of AAA is usually reported between 1.5 - 5\% (e.g. [24] reported 2.2\% in 65-year-old Swedish men; and [25] gave an extrapolation to $3.5 \%$ and $4.5 \%$ at the same age). The age of 85 years was estimated with an extrapolation to $3.5 \%$ on the Czech population aged between 65 and 100 years. A 3.5\% portion in each age group was computed from demographic statistics on length of life obtained from the Czech Statistical Office [26]. The sum of these portions was doubled to account for tortuosity and asymptomatic cases. The result was considered as an estimate of the number of individuals with zero prestretch in the population. Strictly speaking, it is not known how these cases are distributed in the population. However, a conservative estimate of the zero-prestretch age can be obtained when all nonprestretched cases are inserted into the empirical density distribution of age, decreasing from 100 years of age. A conservative estimate means that it does not overestimate aging-induced changes (the loss of prestretch). On the other hand it means that the loss of prestretch may occur earlier, but in average it should not be later. These cases covered the interval between the eighty-fifth year of age and the last age in the statistics (100 years). Thus zero state was prescribed at the age of 85 years.

The same form of the regression model (10) was adopted for prestretch, prestress and the pretension force-age relationship. Equation (10) contains three parameters, $c_{0}, c_{1}$ and $c_{2}$, but the number of free parameters is restricted by the conditions $y(20)=1$ and $y(85)=0$.

$$
y=c_{0}\left(1-c_{1} e^{-c_{2} x}\right)
$$

Note that the regression was performed with normalized data. Taking into account previous observations (Horny et al., 2011, 2012), $\lambda_{\text {AUTOPSY }}=1.4$ was prescribed as the expected value at the age of 20 years, which implies $\varepsilon_{20}=0.4$ as the normalization constant. In case of prestress and pretension force, the normalization constants were considered to be unknown a priori. They were therefore optimized in the regression analysis. 


\section{Results}

The study involved 10 human donors (6 female, 4 male) of infrarenal aorta. Descriptive statistics of the samples are listed in Table 1. Sample ID identifies the gender (M, F) and the age of the donor.

Pretension force, prestress and stress-strain relationships. The experimental records obtained in the elongation of the cylindrical samples are depicted in Fig. 1 and 2. The prestretch measured in the excision of the sample from a body is indicated by a vertical line. The prestress and the pretension force, listed in Table 1, were determined as the position of the intersection in these graphs (mean/SSD - autopsy prestretch 1.13/0.1 pretension force 0.54/0.44 N; and prestress 11.6/13.5 kPa; SSD denotes sample standard deviation). It is clearly visible that younger donors manifest a higher prestretch and pretension force. The mechanical responses also confirmed our presumption that older donors will yield stiffer aortas.

The stress-strain responses were fitted to the limiting chain extensibility model (3). The models correspond well with the observed behavior, see Fig. 3. The estimated parameters are listed in Table 2. The results show that age-dependency is primarily governed by $J_{m}$.

Elastic modulus. The results are listed in Table 2. The mean value estimated in spatial description (i.e. using true stress and deformed cross-sectional area) was 167/210 kPa (mean/SSD).

Correlation with age. All studied quantities showed a correlation with calendar age (Figures 1-5). Fig. 4 displays the results of the regression analysis aimed at the prestretch-age relationship, comparing the current (small) sample with previously observed data. To this end, prediction intervals (PI; 95\% confidence) based on the large sample were added (construction described in Horny et al., 2011). It turns out that the current observations are not exceptional, and may be considered as apposite representatives. The model parameters are cited in the figure legend. In the small data sample, the linear correlation coefficient $R$ was (considering logarithmic transformation of the power law) -0.919 for prestress-age, -0.946 prestretch-age, and -0.800 for pretension force-age dependence. These values are higher than the correlation coefficients (see Table 1) based on raw data (linear relationship between the variables). The $p$-values confirm this result. They particularly suggest that a linear equation is not an efficient description for the prestress-age relationship ( $p_{a}=0.43$ vs. $p_{b}<0.001$ ). Generally, the power law was more successful in describing the age-dependency of primary quantities.

However, this is not the case for the material parameters $\mu$ and $J_{m}$. The statistical analysis results suggest that we should reject the hypothesis that $\mu$ is age dependent (using both linear and power law relationships, see Table 2). In the case of $J_{m}$, which correlates significantly with age, a linear 
description is more successful. Bearing this in mind, we went on to investigate whether $J_{m}$ alone could explain the age-dependent variability in the stress-strain data. $\mu$ was fixed to its mean value (20.24 $\mathrm{kPa}$ ), and each curve was fitted again. The results showed (Fig. 6) that this approach is capable of expressing the character of the aging effect. However, the data for younger donors is somewhat overestimated at the initial deformations.

Longitudinal elastic modulus $\underline{c}_{z z z z}$ (related to autopsy prestretch) did not have as high a correlation with age as prestretch, force and prestress. The data suggests decreasing trend with advancing age $(R=$ $-0.632, p_{R}=0.025$, power law model). Nevertheless, neither $p_{a}$ nor $p_{b}$ did not reach significance.

Normalized models. Equation (10) was used to model age-related changes of the prestretch, prestress and pretension force considering not only decreasing trend but also the state of zero longitudinal pretension. The correlation coefficient (after transformation to the linear problem) had values of -0.863 for prestretch in the large sample, and -0.910 for prestretch, -0.914 for prestress, and 0.792 for pretension force in the small sample. The parameters of the model are listed in the legend of Fig. 5. In Fig. 4, a simple power law model (based on the small data sample; red curve) is compared with (10) for the large data sample (thick black curve). It is clearly shown that at the intersection of the domains of observation the two models give similar predictions. Computation of the $p$-values for the regression given by (10) has therefore been omitted. Note that although the simple power law gives similar predictions, it does not hold outside this domain. They differ significantly in their asymptotic behavior, because the power law $\left(y=a x^{b}\right)$ cannot predict the loss of longitudinal pre-stress and pretension force since it approaches zero at infinity. Contrastingly, equation (10) is able to describe this property and was employed for this purpose.

\section{Discussion}

Arteriosclerosis of elastic arteries is one of the most apparent manifestations of advanced age in the cardiovascular system. Elastic arteries enlarge their diameter and stiffen [27-29]. This process can be attributed to several mechanobiological events at the molecular and cellular length scale. Among others, fragmentation and thinning of elastin lamellae [30-32], which is coincident with increased matrix proteinase activity [30,33], occurs in aging. This affects the arterial elasticity, since elastin is the dominant load-bearing component under physiological loadings (less than $10 \%$ of collagen fibers are engaged in a physiological situation [34]). Increased calcium deposition is also usually observed (medial elasto-calcinosis [35]). It can be accompanied by a change in cellular phenotype (transdifferentation of smooth muscle cells to bone-like cells [36]). Advanced glycation end-products also contribute to stiffening cross-linking collagens and also elastin [37]. These changes may be viewed as the source of the mechanobiological perturbations which initialize remodeling and 
Horny L, Adamek T, Zitny R (2013) Age-related changes in longitudinal prestress in human abdominal aorta. Arch Appl Mech 83(6):875-88. doi: 10.1007/s00419-012-0723-4 MANUSCRIPT VERSION

adaptation. Remodeling and mentioned degradative processes result in an enlarged diameter, a stiffened mechanical response, and loss of pretension. Our previous studies showed age-related changes of the axial prestrain in detail $[1,18,19]$. However, they did not address the question of prestress.

Prestretch, prestress, pretension force. The study confirmed that longitudinal prestretch decreases with advanced age, and the same finding was newly recorded for prestress and pretension force. The highest force $(1.63 \mathrm{~N})$ was obtained for a 29 -year-old female, and the lowest force $(0.14 \mathrm{~N})$ was for a man aged 61 . These samples also showed the highest and lowest prestretch and prestress (prestress $/$ prestretch $=45 \mathrm{kPa} / 1.32$ and $2.3 \mathrm{kPa} / 1.03$ ).

The observed mean values for prestress and pretension force $(11.6 \mathrm{kPa} / 0.54 \mathrm{~N})$ are somewhat smaller than the values reported in the literature. $50 \mathrm{kPa}$ and $1 \mathrm{~N}$ were estimated by Han and Fung [17] for porcine and canine aortas, while the estimate based on Dobrin and Doyle [3], $0.01 \mathrm{~N} / \mathrm{kg}$, gives 0.75 $\mathrm{N}$, taking $75 \mathrm{~kg}$ as the representative human body weight. The difference may be attributed to interspecies differences (Dobrin and Doyle involve canine specimens). In our specific case, lower values for the observed prestress and pretension force are affected by the age distribution in the sample (47/12; mean/SSD). However, it is more interesting to study an aged population because the results in $[3,17]$ can serve as estimates for physiologically optimal conditions.

Elastic modulus. The hyperelastic limiting chain extensibility model (3) was adopted to elucidate whether decreasing pretension is accompanied by decreased or increased stiffness. It was found that the human abdominal aorta does not stiffen longitudinally in its prestress-prestrain state (elongated under zero internal pressure). The correlation coefficient is negative and significance was attained in both linear and power regression model $\left(R=-0.566, p_{R}=0.04 ; R=-0.632, p_{R}=0.03\right)$, but neither linear nor power dependence on age seems to be suitable because $p_{a}=p_{b}=0.1$.

It is widely accepted that elastin is the most important load-bearing component in the vascular wall in initial and physiological loading [21-23,27]. Moreover, elongations of cylindrical samples (at zero transmural pressure) are accompanied by decreasing radius (compressive circumferential strains). Hence, we can assume that collagen fibers (the main load-bearing component at supraphysiological loading), which are oriented predominantly in the circumferential direction [38], were not operative during extensions up to $\lambda_{\text {AUTOPSY }}$ (neglecting load-carrying capacity other than tensile stiffness). In this case, the results may be attributed to the elastin. Non-increasing stiffness of the elastin is in agreement with [39].

Our conclusion about non-increasing stiffness is valid, however, only for strain-state corresponding to the longitudinal prestretch (at zero pressure). Figs. 1-3 and 6 show clearly that a loading exceeding $\lambda_{\text {AUTOPSY }}$ generates steeply increasing stress-strain curves, where aging is manifested by greater steepness of the curves (i.e. greater stiffness). 
Constitutive parameters. Parameter $\mu$, which is responsible for the stiffness at infinitesimal strains, showed no demonstrable correlation with age (Table 2). This suggests that almost all agerelated variability may be attributed to $J_{m}$, which is consistent with its interpretation as a measure of the limiting extensibility [21]. Measured by $J_{m}$, the extensibility decreases with age; $R=-0.818$ $\left(p_{R}<0.002\right.$ raw data). Numerical values of $J_{m}$ found in our study $\left(J_{m} \in[0.0970 ; 0.7592]\right.$, see Table 2$)$ correspond well with estimates known from the literature (results found in [49,50] satisfy $0<J_{m}<2.5$ with variability coming from age, location in arterial tree, and animal species).

The observed progressive stiffening at deformations exceeding $\lambda_{\text {AUTOPSY }}$ cannot be related only to the elastin, because the extensions are supraphysiological. It is known that the adventitia (the outermost layer of the artery wall) contains helically oriented collagen fibers $[22,40]$. We interpret the stiffening (when $\lambda>\lambda_{\text {AUTOPSY) }}$ ) as their non-negligible engagement to the load-carrying process which reflects higher degree of collagen cross-linking and other aging-induced changes in the collagens. It should be noted that the application of equation (9) when $\lambda>\lambda_{\text {AUTOPSY }}$ gives a stiffness value which cannot be considered as isotropic.

Normalized models of aging-induced changes. To the best of our knowledge this study is the first to attempt to estimate the age corresponding to the total loss of longitudinal pretension. Apart from our data (two individuals with $\lambda_{\text {AUTOPSY }}=1$ aged 83 and 95 years in the large sample), total loss of the pretension was experimentally confirmed in aged human iliac arteries (one individual aged 81 [4]) and for human thoracic aorta (two individuals aged 85 and 88 years [16]). Both these publications even reported $\lambda_{\text {AUTOPSY }}<1$ (longitudinal pre-compression). Bearing this in mind, and considering relatively frequent diseases such as aneurysms and tortuosity, we prescribed 85 years of age as the time when total loss of pretension may be expected. Figure 4 shows that the model incorporating this presumption gives some outliers. Although one could suggest shifting the loss of prestretch to a higher age (PI would be enlarged in this way), we note that our statistics is affected by the "sampling method". Our studies were primarily aimed at a study of individuals with well-defined prestretch, and aneurysmatic and tortuous aortas were excluded. Since known data gives 83, 95 (our sample), 85, 88 and 81 (literature), we hold the prescribed age (85 years) for loss of pretension as the first approximation. Future studies will probably refine our result.

In fact, it is not known whether loss of prestress is an inevitable consequence of aging or a "rare" pathological condition. The power law regression models for prestress-age and pretension force-age are therefore also presented (Table 1). These models reflect the situation when the hypothesis of inevitable loss of prestress is not accepted. These models also decrease progressively with age but they only asymptotically approach zero. It is worth noting that very little is known about longitudinal prestretch in aneurysmatic aortas [41]. We presumed that the development of an aneurysm is 
accompanied by total loss of the pretension. Future studies, however could show that there is some threshold in the longitudinal force (likely very small but non-zero) sustained by an artery below which aneurysm formation can initiate. In this situation, an asymptotical approach to zero would be more appropriate.

Equation (10) for a normalized description of aging was chosen with respect to a phenomenological analogy with a discharging capacitor and Newton's law of cooling. This means that the governing equation can be written in the form $d y / d t=c_{2}\left(c_{0}-y\right)$. We note that negative values of $c_{0}$ (see the caption of Fig. 5) are the consequence of the prescribed zero-prestretch age.

A similar idea, a simulation employing a time rate of the longitudinal prestretch, was recently presented by Tsamis et al. [42]. The authors hypothesized that aging-induced damage to elastin (fragmentation and thinning) initializes a remodeling of the arterial geometry. This damage can result from an elastolysis with a concurrent contribution from material fatigue due to the remarkable number of pressure pulse cycles that elastic arteries have to sustain throughout the entire lifespan $[34,43]$. Since longitudinal prestretch is mainly carried by elastin $[7,12,14]$ we hypothesize that our observation of decreasing longitudinal prestretch is also a consequence of aging-induced damage to the elastin.

The decrease in longitudinal prestress can be explained by an age-related increase in the crosssectional area (observed in our study and in many others, Table 1). Increased cross-section area gives lower prestress. The lower prestress would certainly induce a decrease in the prestretch. But what is the source of the decrease in the pretension force observed in our experiments? The paradigm states that an artery is remodeled to reduce the stress, not the force.

This force can, however, be released in permanent deformation of the elastin membranes (disruption, fragmentation), which is then macroscopically observed as a lengthened ex situ geometry [18]. This is in accordance with the hypothesis that decreased longitudinal prestretch originates in aging-induced damage to elastin. However, at current state of knowledge it is not possible to say that this is the sole cause of the observed phenomena. For instance, the role of the perivascular tissue is unclear (we suppose that during the developmental period the pretension force is imprinted on external bonds which hold the aorta in its position). Perivascular-vascular interaction, sometimes called tethering, is frequently neglected in the literature. Thus we have no evidence on how aging affects this bond. Theoretically, it is possible that there are some age-related changes to this interaction which could contribute to a decrease of the longitudinal preload.

Estimate of consequences for the in vivo state. In experimental (ex vivo but in situ) inflation of a canine carotid artery, Dobrin and Doyle [3] found that the total longitudinal stress in the artery wall changes only slightly. This was due to a simultaneous decrease in the pretension force (to mimic in vivo conditions they held a constant longitudinal prestretch during pressurization) and an increase in the force induced by internal pressure $P$ acting at the end of a vessel. The equation $\sigma_{z z}=\sigma_{z z P}+\sigma_{z z F}=$ $P r_{i}^{2} /\left(r_{o}^{2}-r_{i}^{2}\right)+F /\left(\pi\left(r_{o}^{2}-r_{i}^{2}\right)\right)$ describes this situation; $r_{o}$ and $r_{i}$ denote the outer and inner deformed 
radius. We did not perform inflation-extension experiments, and thus cannot describe specific values of the total longitudinal stress (we will pursue this objective in future work). The equation, however, enables the situation to be discussed qualitatively. The initial value (at $P=0$ ) of $F$ corresponds just to our pretension force. Considering that the pretension force has been proven to be decreasing during aging, we find that the term $\sigma_{z z F}=F /\left(\pi\left(r_{o}^{2}-r_{i}^{2}\right)\right)$ can approach 0 before the pressurization because the aged arteries are not prestretched. In such a case, however, a balance between increment of $\sigma_{z z P}$ and decrement of $\sigma_{z z F}$ would be lost. Then, the pattern of the longitudinal stress would be governed by $\sigma_{z z P}$ $=P r_{i}^{2} /\left(r_{o}^{2}-r_{i}^{2}\right)$ and the variations due to the pressure cycle would appear. The variation of the longitudinal stress could be the reason for the longitudinal oscillations observed in vivo by modern imaging techniques [44].

\section{Conclusion}

We conclude that the longitudinal pretension force and the prestress, as well as the longitudinal prestretch, decrease significantly during aging. The longitudinal stiffness related to the prestretch of a human abdominal aorta observed in autopsies did not increase with advancing age. However, the overall mechanical response to longitudinal extension was characterized by significant stiffening. Constitutive modeling suggested that limiting chain extensibility is a concept suitable for describing the aging effect.

The present study has also revealed that further research is needed to elucidate to what extent the age-related decrease in longitudinal prestress, prestrain and related force is induced by remodeling, by damage to the elastin and by changes in the perivascular-vascular tissue interaction.

The raw data collected during autopsy measurements has been added to the online supplement. They can be helpful in an in vivo determination of constitutive parameters [45-48]. This promising procedure has to presume some reference and intermediate geometry and related boundary conditions (prestrain and prestress). Prestrain and prestress cannot, however, be accessed in living persons. They therefore have to be estimated in ex vivo studies.

\section{Conflict of interest statement}

The authors declare no conflict of interests.

\section{Acknowledgement}

This work has been supported by the Czech Technical University in Prague under project SGS10/247/OHK2/3T/12, and Czech Ministry of Health project NT 13302, and by Technology agency of the Czech Republic in the project TA 01010185. 
Horny L, Adamek T, Zitny R (2013) Age-related changes in longitudinal prestress in human abdominal aorta. Arch Appl Mech 83(6):875-88. doi: 10.1007/s00419-012-0723-4 MANUSCRIPT VERSION

\section{References}

[1] Horny L, Adamek T, Gultova E, Zitny R, Vesely J, Chlup H, Konvickova S (2011) Correlations between age, prestrain, diameter and atherosclerosis in the male abdominal aorta. J Mech Behav Biomed Mater 4:2128-2132. doi: 10.1016/j.jmbbm.2011.07.011

[2] Learoyd BM, Taylor MG (1966) Alterations with age in the viscoelastic properties of human arterial walls. Circ Res 18:278-292.

[3] Dobrin PB, Doyle JM (1970) Vascular smooth muscle and the anisotropy of dog carotid artery. Circ Res 27:105-119.

[4] Schulze-Bauer CAJ, Morth C, Holzapfel GA (2003) Passive biaxial mechanical response of aged human iliac arteries. J Biomech Eng 125:395-406. doi: 10.1115/1.1574331

[5] Sommer G, Regitnig P, Költringer L, Holzapfel GA (2010) Biaxial mechanical properties of intact and layer-disected human carotid arteries at physiological and supraphysiological loadings. Am J Physiol - Heart Circ Physiol 298:898-912. doi: 10.1152/ajpheart.00378.2009

[6] Han HC, Ku DN, Vito RP (2003) Arterial wall adaptation under elevated longitudinal stretch in organ culture. Ann Biomed Eng 31:403-411. doi: 10.1114/1.1561291

[7] Humphrey JD, Eberth JF, Dye WW, Gleason RL (2009) Fundamental role of axial stress in compensatory adaptations by arteries. J Biomech 42:1-8. doi: 10.1016/j.jbiomech.2008.11.011

[8] Jackson ZS, Gotlieb AI, Langille BL (2002) Wall tissue remodeling regulates longitudinal tension in arteries. Circ Res 90:918-925. doi: 10.1161/01.RES.0000016481.87703.CC

[9] Jackson ZS, Dajnowiec D, Gotlieb AI, Langille BL (2005) Partial off-loading of longitudinal tension induces arterial tortuosity. Arterioscler Thromb Vasc Biol 25:957-962. doi: 10.1161/01.ATV.0000161277. 46464.11

[10] Lee Y-U, Drury-Stewart D, Vito RP, Han H-C (2008) Morphologic adaptation of arterial endothelial cells to longitudinal stretch in organ culture. J Biomech 41:3274-3277. doi: 10.1016/j.jbiomech.2008.08.016

[11] Davis NP, Han HC, Wayman B, Vito R (2005) Sustained axial loading lengthens arteries in organ culture. Ann Biomed Eng 33:867-877. doi: 10.1007/s10439-005-3488-x

[12] Dobrin PB, Schwarcz TH, Mirkvicka R (1990) Longitudinal retractive force in pressurized dog and human arteries. J Surg Res 48:116-120. doi: 10.1016/0022-4804(90)90202-D

[13] Lee AY, Han B, Lamm SD, Fierro CA, Han H-C (2012) Effects of elastin degradation and surrounding matrix support on artery stability. Am J Physiol - Heart Circ Physiol 302:873-884. doi: 10.1152/ajpheart.00463. 2011

[14] Carta L, Wagenseil JE, Knutsen RH, Mariko B, Faury G, Davis EC et al. (2009) Discrete contributions of elastic fiber components to arterial development and mechanical compliance. Arterioscler Thromb Vasc Biol 29:2083-2089. doi: 10.1161/ATVBAHA.109.193227

[15] Wagenseil JE, Mecham RP (2012) Elastin in large artery stiffness and hypertension. J Cardiovasc Trans Res 5:264-273. doi: 10.1007/s12265-012-9349-8

[16] Langewouters GJ, Wesseling KH, Goedhard WJA (1984) The static elastic properties of 45 human thoracic and 20 abdominal aortas in vitro and the parameters of a new model. J Biomech 17:425-435.

[17] Han HC, Fung YC (1995) Longitudinal strain of canine and porcine aortas. J Biomech 28:637-641. doi: 10.1016/0021-9290(94)00091-H

[18] Horny L, Adamek T, Chlup H, Zitny R (2012) Age estimation based on a combined arteriosclerotic index. Int J Leg Med 126:321-326. doi: 10.1007/s00414-011-0653-7

[19] Horny L, Adamek T, Vesely J, Chlup H, Zitny R, Konvickova S (2012) Age-related distribution of longitudinal pre-strain in abdominal aorta with emphasis on forensic application. Forensic Sci Int 214:18-22. doi: 10.1016/j.forsciint.2011.07.007

[20] Gent AN (1996) A new constitutive relation for rubber. Rubber Chem Technol 69:59-61.

[21] Ogden RW, Saccomandi G (2007) Introducing mesoscopic information into constitutive equations for arterial walls. Biomech Model Mechanobiol 6:333-344. doi: 10.1007/s10237-006-0064-8

[22] Holzapfel GA, Gasser TC, Ogden RW (2000) A new constitutive framework for arterial wall mechanics and a comparative study of material models. J Elast 61:1-48. doi: 10.1023/A:1010835316564

[23] Watton PN, Ventikos Y, Holzapfel GA (2009) Modelling the mechanical response of elastin for arterial tissue. J Biomech 42:1320-1325. doi:10.1016/j.biomech.2009.03.012

[24] Svensjö S, Björck M, Gürtelschmid M, Djavani Gidlund K, Hellberg A, Wanhainen A (2011) Low prevalence of abdominal aortic aneurysm among 65-year-old swedish men indicates a change in the epidemiology of the disease. Circulation 124:1118-1123. doi: 10.1161/CIRCULATIONAHA.111.030379

[25] Collective of authors (2001) A comparative study of the prevalence of abdominal aortic aneurysms in the United Kingdom, Denmark, and Australia. J Med Screen 8:46-50. doi: 10.1136/jms.8.1.46 
Horny L, Adamek T, Zitny R (2013) Age-related changes in longitudinal prestress in human abdominal aorta. Arch Appl Mech 83(6):875-88. doi: 10.1007/s00419-012-0723-4 MANUSCRIPT VERSION

[26] Czech Statistical Office (2011) Annual demographical report. http://www.czso.cz/csu/ 2011edicniplan.nsf/publ/4003-11-r_2011

[27] Greenwald SE (2007) Ageing of the conduit arteries. J Pathol 211:157-172. doi: 10.1002/path.2101

[28] O'Rourke MF, Hashimoto J (2007) Mechanical Factors in Arterial Aging: A Clinical Perspective. J Am Coll Cardiol 50:1-13. doi: 10.1016/j.jacc.2006.12.050

[29] McEniery CM, Wilkinson IB, Avolio AP (2007) Age, hypertension and arterial function. Clin Exp Pharmacol Physiol 34:665-671. doi: 10.1111/j.1440-1681.2007.04657.x

[30] Arribas SM, Hinek A, González MC (2006) Elastic fibers and vascular structure in hypertension. Pharmacol Therap 111:771-791. doi: 10.1016/j.pharmthera.2005.12.003

[31] Avolio A, Jones D, Tafazzoli-Shadpour M (1998) Quantification of alternations in structure and function of elastin in the arterial media. Hypertension 32:170-175.

[32] Fonck E, Feigl GG, Fasel J, Sage D, Unser M, Rüfenacht DA, Stergiopulos N (2009) Effect of aging on elastin functionality in human cerebral arteries. Stroke 40:2552-2556. doi: 10.1161/strokeaha.108.528091

[33] Jacob MP (2003) Extracellular matrix remodeling and matrix metalloproteinases in the vascular wall during aging and in pathological conditions. Biomed Pharmacother 57:195-202. doi: 10.1016/S07533322(03)00065-9

[34] Greenwald SE, Moore JE, Rachev A, Kane TP, Meister JJ (1997) Experimental investigation of the distribution of residual strains in the artery wall. J Biomech Eng 119:438-444. doi: 10.1115/1.2798291

[35] Atkinson J (2008) Age-related medial elastocalcinosis in arteries: Mechanisms, animal models, and physiological consequences. J Appl Physiol 105:1643-1651. doi: 10.1152/japplphysiol.90476.2008

[36] Persy V, D'Haese P (2009) Vascular calcification and bone disease: the calcification paradox. Trends Mol Med 15:405-416. doi: 10.1016/j.molmed.2009.07.001

[37] Konova E, Baydanoff S, Atanasova M, Velkova A (2004) Age-related changes in the glycation of human aortic elastin. Exp Gerontol 39:249-254. doi: 10.1016/j.exger.2003.10.003

[38] Haskett D, Johnson G, Zhou A, Utzinger U, Vande Geest J (2010) Microstructural and biomechanical alternations of the human aorta as a function of age and location. Biomech Model Mechanobiol 9:725-736. doi: 10.1007/s10237-010-0209-7

[39] Wuyts FL, Vanhuyse VJ, Langewouters GJ, Decraemer WF, Raman ER, Buyle S (1995) Elastic properties of human aortas in relation to age and atherosclerosis: A structural model. Phys Med Biol 40:15771597.

[40] Gasser TC, Ogden RW, Holzapfel GA (2006) Hyperelastic modelling of arterial layers with distributed collagen fibre orientations J Roy Soc Interface 3:15-35. doi: 10.1098/rsif.2005.0073

[41] Humphrey JD, Holzapfel GA (2012) Mechanics, mechanobiology, and modeling of human abdominal aorta and aneurysms. J Biomech 45:805-814. doi: 10.1016/j.jbiomech.2011.11.021

[42] Tsamis A, Rachev A, Stergiopulos N (2011) A constituent-based model of age-related changes in conduit arteries. Am J Physiol - Heart Circ Physiol 301:1286-1301. doi: 10.1152/ajpheart.00570.2010

[43] Lillie MA, Gosline JM (2007) Limits to the durability of arterial elastic tissue. Biomaterials 28:20212031. doi: 10.1016/j.biomaterials.2007.01.016

[44] Cinthio M, Ahlgren AR, Bergkvist J, Jansson T, Persson HW, Lindstrom K (2006) Longitudinal movements and resulting shear strain of the arterial wall. Am J Physiol - Heart Circ Physiol 291:394-402. doi: 10.1152/ajpheart.00988.2005

[45] Âstrand H, Stålhand J, Karlsson J, Karlsson M, Sonesson B, Länne T (2011) In vivo estimation of the contribution of elastin and collagen to the mechanical properties in the human abdominal aorta: Effect of age and sex. J Appl Physiol 110:176-187. doi: 10.1152/japplphysiol.00579.2010

[46] Masson I, Beaussier H, Boutouyrie P, Laurent S, Humphrey JD, Zidi M (2011) Carotid artery mechanical properties and stresses quantified using in vivo data from normotensive and hypertensive humans. Biomech Model Mechanobiol 10:867-882. doi: 10.1007/s10237-010-0279-6

[47] Schulze-Bauer CAJ, Holzapfel GA (2003) Determination of constitutive equations for human arteries from clinical data. J Biomech 36:165-169. doi: 10.1016/S0021-9290(02)00367-6

[48] Stalhand J (2009) Determination of human arterial wall parameters from clinical data. Biomech Model Mechanobiol 8:141-148. doi: 10.1007/s10237-008-0124-3

[49] Horgan CO, Saccomandi G (2003) A description of arterial wall mechanics using limiting chain extensibility constitutive models. Biomech Model Mechanobiol 1:251-266. doi: 10.1007/s10237-002-0022-z

[50] Destrade M, Ní Annaidh A, Coman CD (2009) Bending instabilities of soft biological tissues. Int J Solids Struct 46:4322-4330. doi: 10.1016/j.ijsolstr.2009.08.017 


\section{Tables}

Table 1 Data summary. The table summarizes measured quantities in the form mean/estimation of uncertainty (if available). The code indicates gender and age [years]. The circumference in the reference configuration, $C$, was obtained as the length of a ring cut from the specimen (determined via image processing). The thickness in the reference configuration, $T$, was measured by a caliper (five times and then averaged). The reference cross-section area, $S$, was obtained as $S=C \cdot T$. PMI denotes the time interval between death and the experiment. ATH quantifies the degree of atherosclerosis examined by the pathologist; 0 - intact artery and fatty streaks; 1 - fibro-fatty plaques; 2 - advanced plaques; 3 - calcified plaques; 4 - ruptured plaques (Kumar et al., 2010). Measurement uncertainties were estimated by means of the sample standard deviation (SSD) for $C, T, S$; in the case of prestress, it is the mean of SSD for the neighboring stresses determined in the elongation test under the strain corresponding to the prestretch; and in case of the prestretch, it is the resolution unit/L. $R$ denotes linear correlation coefficient. It is supplemented with $p$-value $p_{R}$ and $p_{a}\left(p_{R}-\right.$ the null hypothesis $R=0$ will be rejected if the significance level $\alpha$ is higher than $p_{R} ; p_{a}$ - the null hypothesis $a=0$ (related to the regression equation $y=a x+b$ ) will be rejected if the significance level $\alpha$ is higher than $p_{a}$. We note that in the "logarithmic data" row all quantities are computed after logarithmic transformation, and $p$-value $p_{b}$ is related to the hypothesis $b=0$ in $\ln (y)=\ln (a)+b \ln (x)\left(y=a x^{b}\right)$. The row denoted Mean contains averaged mean values and SSD of the mean values listed in the column above. ATH was not considered in the regression analysis due to its rather ordinal-variable nature.

\begin{tabular}{|c|c|c|c|c|c|c|c|c|}
\hline Code & $\begin{array}{c}C \\
{[\mathrm{~mm}]}\end{array}$ & $\begin{array}{c}T \\
{[\mathrm{~mm}]}\end{array}$ & $\begin{array}{c}S \\
{\left[\mathrm{~mm}^{2}\right]}\end{array}$ & $\begin{array}{l}\text { PMI } \\
\text { [hour] }\end{array}$ & $\begin{array}{c}\mathrm{ATH} \\
{[-]}\end{array}$ & $\begin{array}{c}\text { Prestretch } \\
{[1]}\end{array}$ & $\begin{array}{c}\text { Prestress } \\
{[\mathrm{kPa}]}\end{array}$ & $\begin{array}{c}\text { Pretension } \\
\text { force }[\mathrm{N}]\end{array}$ \\
\hline M26 & $34.9 / 1.7$ & $1.41 / 0.09$ & $49.1 / 5.6$ & 32 & 0 & $1.31 / 0.01$ & $25.5 / 3.5$ & 0.96 \\
\hline M52 & $43.0 / 1.0$ & $1.60 / 0.15$ & $68.8 / 8.1$ & 26 & 1 & $1.09 / 0.01$ & $5.6 / 0.8$ & 0.35 \\
\hline M58 & $48.5 / 1.7$ & $1.91 / 0.36$ & $92.4 / 20.6$ & 80 & 2 & $1.10 / 0.01$ & $6.0 / 1.5$ & 0.52 \\
\hline M61 & 43/- & $1.46 / 0.22$ & $62.7 / 9.4$ & 80 & 1 & $1.03 / 0.01$ & $2.3 / 0.4$ & 0.14 \\
\hline F29 & $35.1 / 0.9$ & $1.38 / 0.26$ & $48.4 / 10.4$ & 99 & 0 & $1.32 / 0.02$ & $45.0 / 10.6$ & 1.63 \\
\hline F38 & $32.7 / 0.6$ & $1.35 / 0.09$ & $44.2 / 3.9$ & 67 & 0 & $1.16 / 0.01$ & $10.5 / 1.2$ & 0.40 \\
\hline F47 & 36/- & $1.95 / 0.23$ & $70.1 / 8.3$ & 50 & 2 & $1.07 / 0.01$ & $4.5 / 0.6$ & 0.30 \\
\hline F48 & $36.5 / 0.5$ & $1.62 / 0.17$ & $59.3 / 6.9$ & 25 & 1 & $1.09 / 0.02$ & $6.0 / 0.7$ & 0.33 \\
\hline F53 & $40.5 / 1.5$ & $1.56 / 0.23$ & $63.3 / 11.9$ & 16 & 2 & $1.08 / 0.01$ & $7.5 / 1.5$ & 0.44 \\
\hline F58 & $48.5 / 0.5$ & $2.29 / 0.37$ & $110 / 17.7$ & 29 & 4 & $1.09 / 0.01$ & $3.3 / 0.7$ & 0.33 \\
\hline $\begin{array}{c}\text { Mean/SSD } \\
\text { (Median) }\end{array}$ & $39.9 / 5.7$ & $1.65 / 0.30$ & $63.8 / 25.2$ & $50 / 29$ & (1) & $1.13 / 0.10$ & $11.6 / 13.5$ & $0.54 / 0.44$ \\
\hline $\begin{array}{c}\text { Correlation } \\
\text { with age } \\
\text { (row data) } \\
R\end{array}$ & 0.802 & 0.567 & 0.768 & - & 0.711 & -0.920 & -0.829 & -0.767 \\
\hline & $\begin{array}{c}p_{R}<0.003 \\
p_{a}=0.36\end{array}$ & $\begin{array}{l}p_{R}=0.044 \\
p_{a}=0.016\end{array}$ & $\begin{array}{c}p_{R}<0.005 \\
p_{a}=0.47\end{array}$ & - & $\begin{array}{c}p_{R}<0.011 \\
-\end{array}$ & $\begin{array}{l}p_{R}<0.001 \\
p_{a}<0.001\end{array}$ & $\begin{array}{c}p_{R}<0.002 \\
p_{a}=0.43\end{array}$ & $\begin{array}{l}p_{R}<0.005 \\
p_{a}<0.002\end{array}$ \\
\hline $\begin{array}{c}a \\
b \\
y=a x+b\end{array}$ & $\begin{array}{c}0.3748 \\
22.25\end{array}$ & $\begin{array}{l}0.0141 \\
0.9889\end{array}$ & $\begin{array}{r}1.580 \\
-10.45\end{array}$ & - & - & $\begin{array}{c}-0.0075 \\
1.484\end{array}$ & $\begin{array}{c}-0.9134 \\
54.55\end{array}$ & $\begin{array}{c}-0.0276 \\
1.834\end{array}$ \\
\hline \multirow[t]{2}{*}{$\begin{array}{l}\text { Correlation } \\
\text { with age } \\
\text { (logarithmic } \\
\text { data) } \\
R\end{array}$} & 0.765 & 0.591 & 0.764 & - & - & -0.946 & -0.919 & -0.800 \\
\hline & $\begin{array}{l}p_{R}<0.005 \\
p_{b}<0.001\end{array}$ & $\begin{array}{l}p_{R}<0.036 \\
p_{b}<0.001\end{array}$ & $\begin{array}{l}p_{R}<0.006 \\
p_{b}<0.004\end{array}$ & - & - & $\begin{array}{l}p_{R}<0.001 \\
p_{b}<0.001\end{array}$ & $\begin{array}{l}p_{R}<0.002 \\
p_{b}<0.001\end{array}$ & $\begin{array}{l}p_{R}<0.005 \\
p_{b}<0.008\end{array}$ \\
\hline $\begin{array}{c}a \\
b \\
y=a x^{b}\end{array}$ & $\begin{array}{l}8.4810 \\
0.4041\end{array}$ & $\begin{array}{l}0.4021 \\
0.3693\end{array}$ & $\begin{array}{c}0.5714 \\
1.222\end{array}$ & - & - & $\begin{array}{c}3.180 \\
-0.2713\end{array}$ & $\begin{array}{c}1.227 \cdot 10^{5} \\
-2.496\end{array}$ & $\begin{array}{c}510.9 \\
-1.836\end{array}$ \\
\hline
\end{tabular}


Horny L, Adamek T, Zitny R (2013) Age-related changes in longitudinal prestress in human abdominal aorta. Arch Appl Mech 83(6):875-88. doi: 10.1007/s00419-012-0723-4 MANUSCRIPT VERSION

Table 2 Model parameters $\left(\mu, J_{m}\right)$ and elastic modulus. Elastic modulus and limiting extensibility were estimated twice, with free $\mu$ and with fixed $\mu$, to highlight the ability of $J_{m}$ to capture the effect of aging. $R, p_{R}, p_{a}$ and $p_{b}$ have the same meaning as in Table 1 .

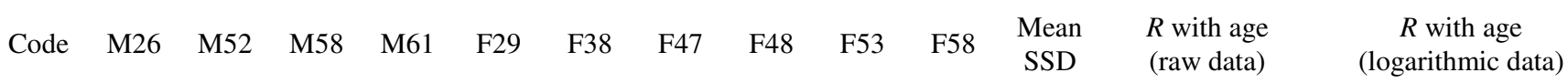

\begin{tabular}{|c|c|c|c|c|c|c|c|c|c|c|c|c|c|c|c|}
\hline $\begin{array}{c}\mu \\
{[\mathrm{kPa}]}\end{array}$ & 19.49 & 22.18 & 13.64 & 29.57 & 14.30 & 19.60 & 22.88 & 23.03 & 27.30 & 10.43 & $\begin{array}{c}20.24 \\
6.07\end{array}$ & 0.200 & $\begin{array}{l}p_{R}=0.29 \\
p_{a}=0.37\end{array}$ & 0.120 & $\begin{array}{l}p_{R}=0.37 \\
p_{b}=0.37\end{array}$ \\
\hline $\begin{array}{c}J_{m} \\
{[1]}\end{array}$ & 0.7592 & 0.3160 & 0.0826 & 0.1982 & 0.3788 & 0.6287 & 0.1196 & 0.2208 & 0.1143 & 0.0970 & $\begin{array}{l}0.2910 \\
0.2349\end{array}$ & -0.818 & $\begin{array}{l}p_{R}<0.002 \\
p_{a}<0.017\end{array}$ & -0.793 & $\begin{array}{l}p_{R}=0.003 \\
p_{b}=0.017\end{array}$ \\
\hline$\frac{\underline{c}_{z z z z}}{[\mathrm{kPa}]}$ & 161.0 & 77.10 & 125.4 & 89.61 & 758.9 & 75.12 & 93.31 & 92.43 & 134.6 & 64.19 & $\begin{array}{l}167.2 \\
210.1\end{array}$ & -0.566 & $\begin{array}{c}p_{R}=0.044 \\
p_{a}=0.10\end{array}$ & -0.632 & $\begin{array}{c}p_{R}=0.025 \\
p_{b}=0.10\end{array}$ \\
\hline $\begin{array}{c}\mu \\
{[\mathrm{kPa}]}\end{array}$ & & & & & 20. & .24 & & & & & - & - & - & - & - \\
\hline $\begin{array}{c}J_{m} \\
{[1]}\end{array}$ & 0.7882 & 0.2851 & 0.1100 & 0.1408 & 0.4883 & 0.6561 & 0.1073 & 0.1976 & 0.0933 & 0.2008 & $\begin{array}{l}0.3068 \\
0.2499\end{array}$ & -0.861 & $\begin{array}{l}p_{R}<0.001 \\
p_{a}<0.008\end{array}$ & -0.823 & $\begin{array}{l}p_{R}<0.002 \\
p_{b}<0.008\end{array}$ \\
\hline$\frac{\underline{c}_{z z z z}}{[\mathrm{kPa}]}$ & 159.3 & 72.14 & 134.4 & 62.32 & 435.6 & 76.32 & 85.39 & 84.99 & 114.3 & 81.48 & $\begin{array}{l}130.6 \\
111.4\end{array}$ & -0.611 & $\begin{array}{l}p_{R}=0.03 \\
p_{a}=0.04\end{array}$ & -0.671 & $\begin{array}{l}p_{R}=0.017 \\
p_{b}=0.039\end{array}$ \\
\hline
\end{tabular}




\section{Figure legends}

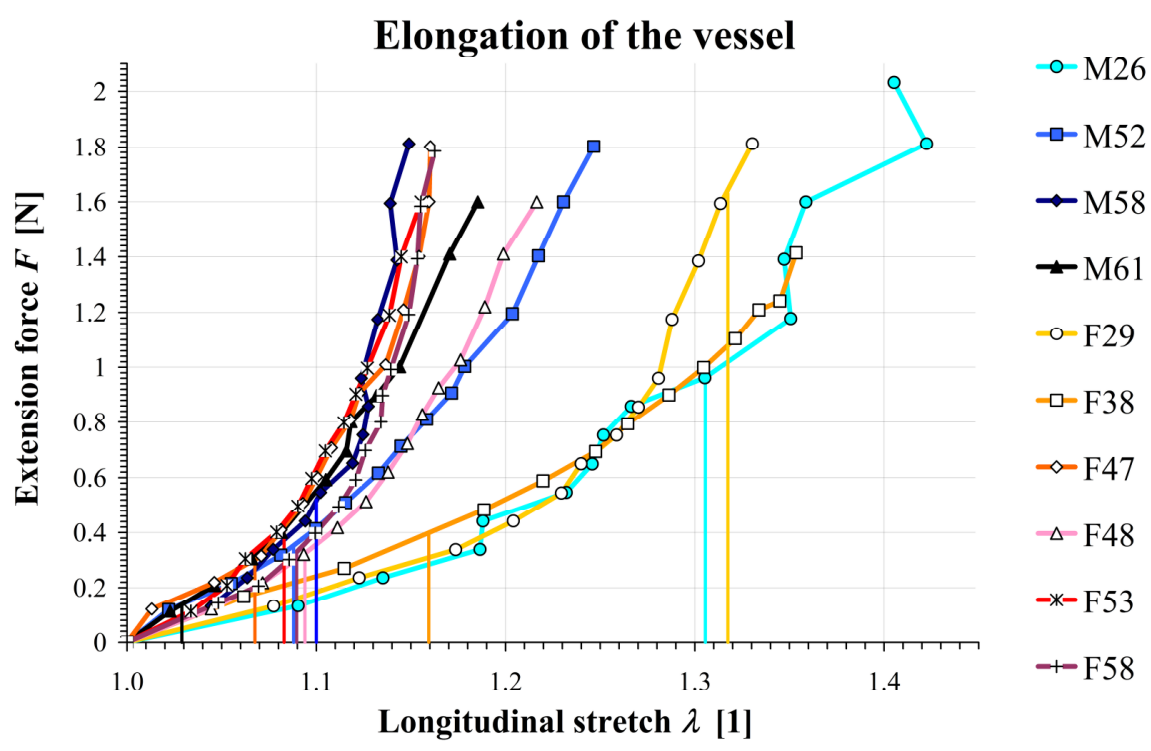

Figure 1 Longitudinal force-stretch diagram obtained during simple elongation of the cylindrical segments of infrarenal aorta. Vertical lines highlight the prestretch. The intersection between the force record and a vertical line determines the presumed axial force acting in the vessel before it is excised. The difference between younger individuals (M26, F29 and F38) and older individuals (M52, M58, M61, F47, F48, F53 and F58) is apparent.

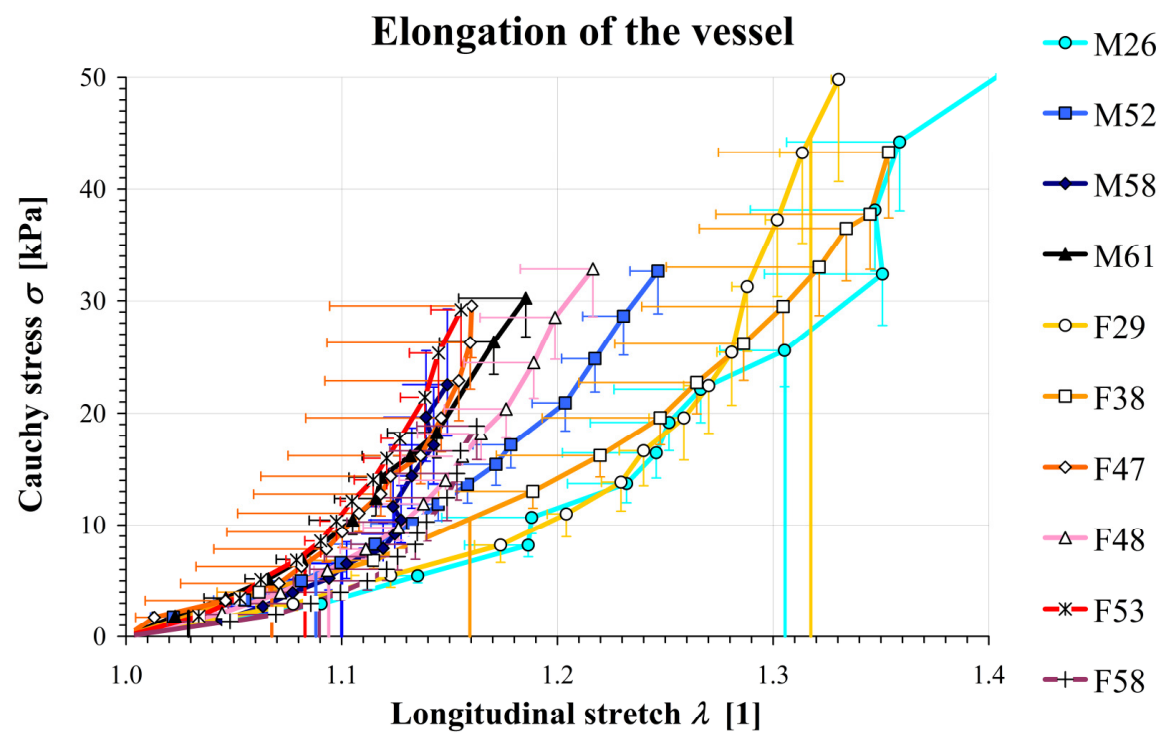

Figure 2 Experimental stress-strain records obtained in the elongation test. The error bars indicate the sample standard deviation. This graph and the following graphs are restricted to $50 \mathrm{kPa}$ in order to display the prestress-prestretch relationship effectively. 


\section{Elongation of the vessel}

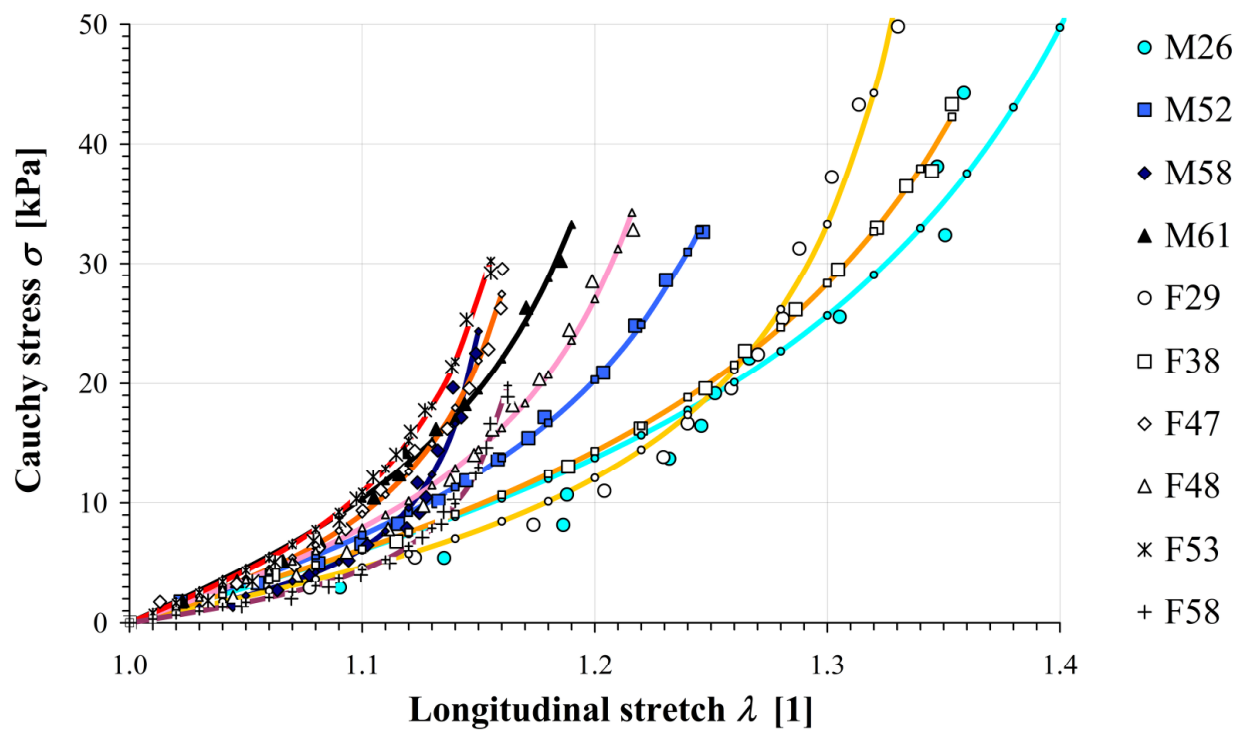

Figure 3 Experiment and model predictions. The parameters of the model are listed in Table 2.

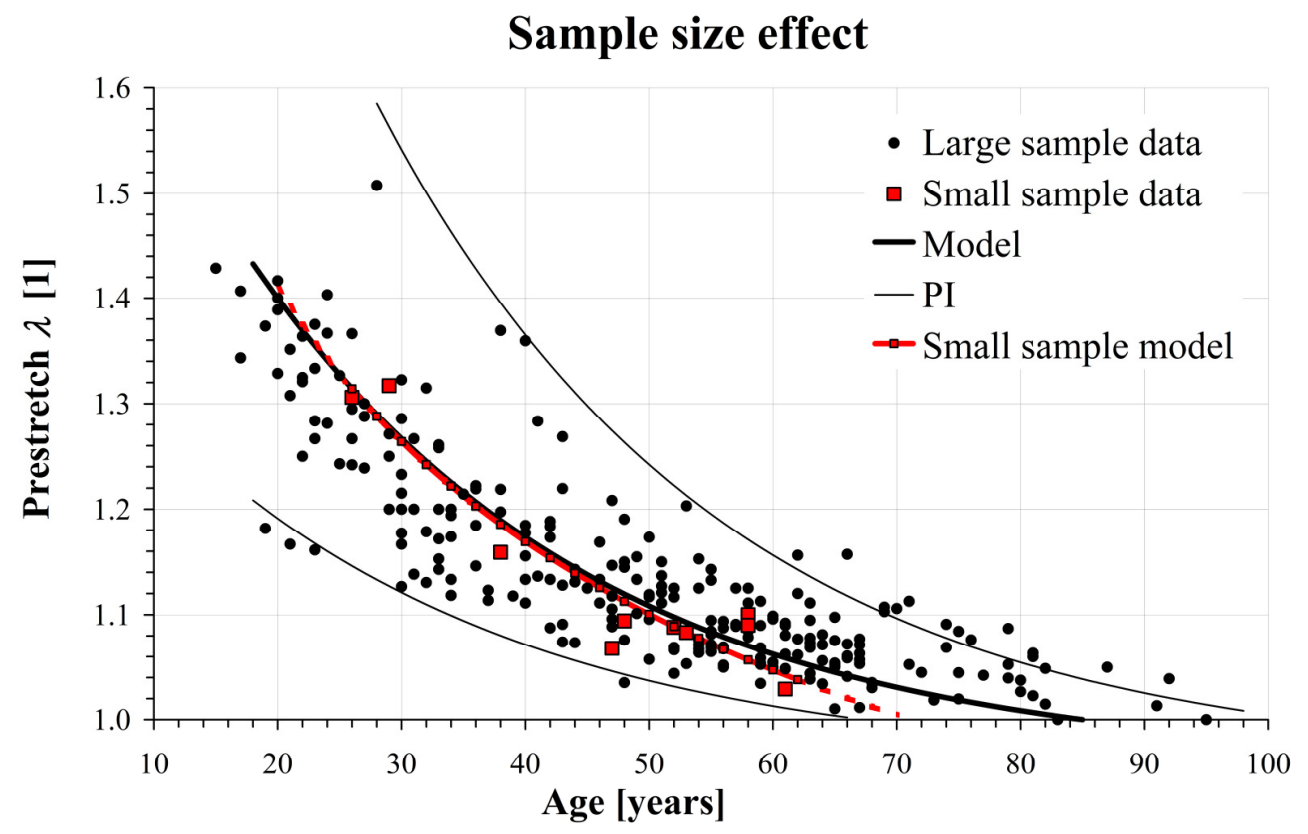

Figure 4 Comparison of data samples. Previously reported data (Horny et al. 2012a) is depicted with black points, current data sample with red boxes. The large sample model is based on equation (10), which gives $\lambda(x)=\varepsilon_{20} c_{0}\left(1-c_{1} e^{-c_{2} x}\right)+1$ with parameters $\varepsilon_{20}=0.4, c_{0}=-0.1082, c_{1}=20.95$, and $c_{2}=0.0358$ ( $x$ denotes age in years). The small sample model is based on the power law $y=a x^{b}$ where $a=3.1804$, $b=-0.2713$. Extrapolation from the observation domain in the small sample model is depicted with a dotted curve. The small sample model approaches $\lambda=1$ at the age of 71 years. 


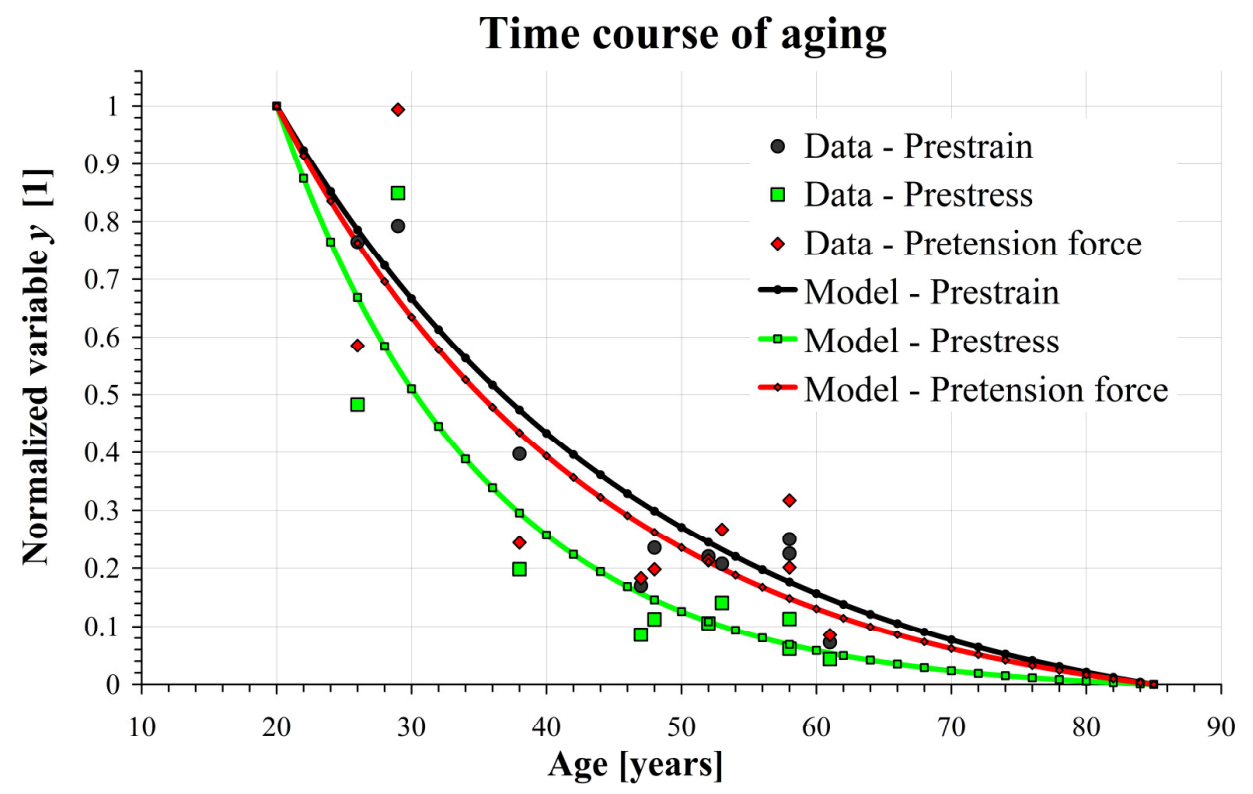

Figure 5 Normalized regression models for the effect of aging. The models are based on the equation $y(x)=c_{0}\left(1-c_{1} e^{-c_{2} x}\right)$. The parameters were estimated as follows: $c_{0}=-0.1082, c_{1}=20.95$, and $c_{2}=$ 0.0358 for normalized prestrain-age, $c_{0}=-0.0139, c_{1}=273.3$ and $c_{2}=0.0660$ for normalized prestress-age, $c_{0}=-0.0711, c_{1}=34.68$, and $c_{2}=-0.0417$ for the normalized pretension force-age relationship. In order to obtain predictions in physical dimensions (non-normalized data), proportionality constants have to be included (they correspond to the values predicted for the age of 20 years); $\varepsilon_{20}=0.4, \sigma_{20}=53 \mathrm{kPa}$, and $F_{20}=1.64 \mathrm{~N}$.

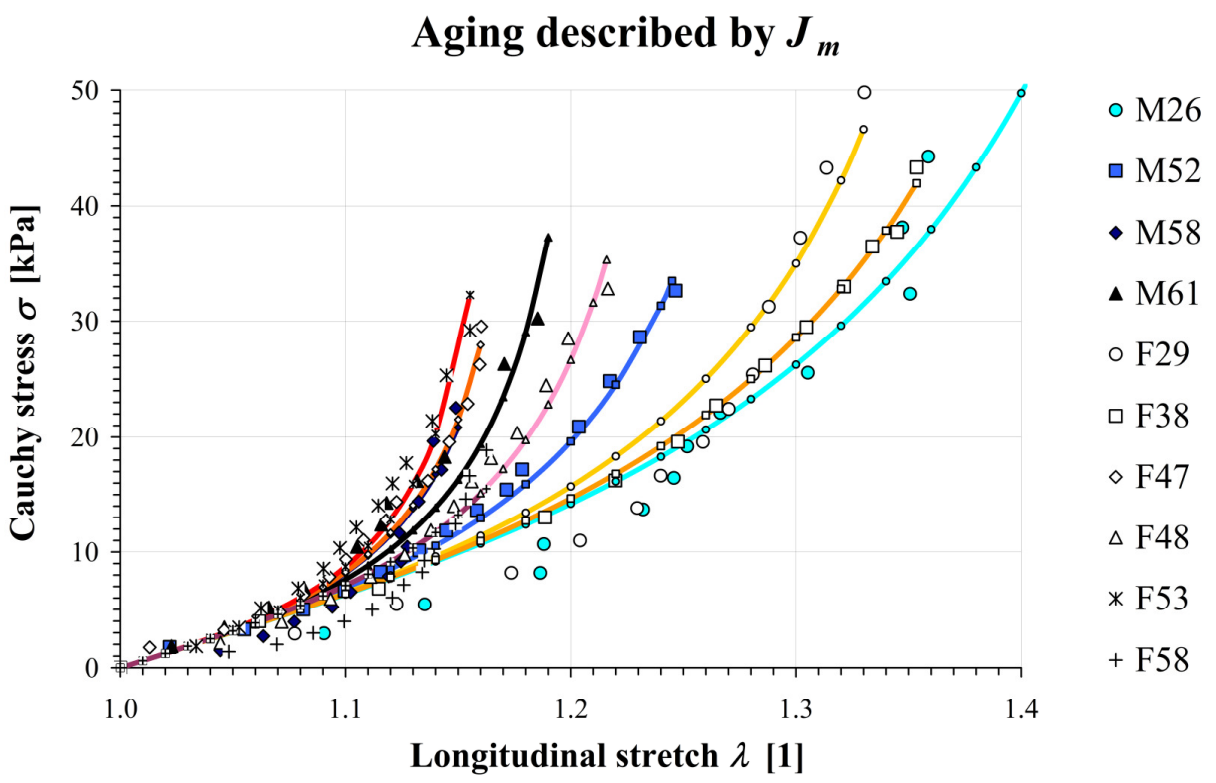

Figure 6 Limiting extensibility reflects the aging effect. Infinitesimal shear modulus $\mu$ was fixed, and all aging variability was left to be explained only by $J_{m}$. The model confirmed its ability to describe aging, though the initial stresses for young donors seem to be rather overestimated (M26, F29, F38). 